\title{
The Role of Positron-Emission
} Tomography/Computed Tomography in Patients with Colorectal Liver Metastases who are Candidates for Liver

\section{Resection: Is it Useful?}

Keywords: ${ }^{18}$ F-fluorodeoxyglucose-positron emission tomography (18-FDG-PET); Colorectal cancer liver metastases; Liver resection

\begin{abstract}
Background: This study aimed to analyze the role of ${ }^{18} \mathrm{~F}$-fluorodeoxyglucose (FDG)-Positron Emission Tomography (PET) as a preoperative diagnostic modality in patients with Colorecta Cancer Liver Metastasis (CRLM) who were candidates for live resection. Oncologists have emphasized the usefulness of FDG-PET/CT. Considering the high cost of PET/CT examinations; it is unclear whether hepatectomy-candidates with CRLM should routinely undergo FDGPET/CT.
\end{abstract}

Methods: In the first term (2002 - 2008), we intended to identify the criteria under which PET was recommended in a retrospective analysis of 50 patients with CRLM. In the second term (2008 - 2013) 30 of 45 patients with CRLM underwent PET/CT by these criteria. The ability of PET/CT to detect extrahepatic lesions was compared with conventional radiologic modalities, such as enhanced CT and Magnetic Resonance Imaging (MRI)

Results: In the first term, all 25 non-PET patients underwent hepatectomy, while hepatectomy was canceled in six of 25 PE patients due to extrahepatic lesions. The prognosis after hepatectomy was similar between the PET and non-PET patients. Since each hepatectomy-canceled patient showed Fong's Clinical Risk Scores (CRS) of three or higher, we determined that the preoperative PET/ CT should be performed when the CRS was greater than or equal to three. In the second term, extrahepatic lesions were identified in four of the 30 PET patients. These lesions were detectable under enhanced multi-detector row CT (MDCT). The lesion detection rate for PET/CT was similar to that of MDCT. Disease quickly recurred after hepatectomy in the PET group (median disease-free duration 10.5 versus 60.0 months) suggesting that preoperative PET/CT did not benefit hepatectomy candidates with CRLM.

Conclusion: In the hepatectomy-candidates with CRLM, PET/CT is not always a necessary diagnostic modality.

\section{Introduction}

Colorectal cancer (CRC) is the third most common cancer and the third leading cause of cancer death in men and women worldwide, with nearly 1.4 million new cases diagnosed in 2012, representing 9.7\% of cancers worldwide [1]. Although CRC is less common in Japan than in Western Europe and the United States, the incidence rate is increasing, with more than 100,000 new cases per year $[2,3]$. The liver is the most common metastatic site of CRC. In about half of CRC patients, the cancer recurs in the liver within 5 years after the initial diagnosis [4]. Although chemotherapy is becoming a more

\section{Journal of} Surgery

\section{Takanori Hiraide $^{1 *}$, Takanori Sakaguchi ${ }^{1}$, Hirotoshi Kikuchi', Makoto Takeda', Yasushi Shibasaki', Yoshifumi Morita', Kazuhiko Fukumoto' ${ }^{2}$, Yasuo Takehara $^{3}$, Shohachi Suzuki ${ }^{2}$, Harumi Sakahara ${ }^{3}$ and Hiroyuki Konno ${ }^{4}$}

${ }^{\prime}$ Second Department of Surgery, Hamamatsu University School of Medicine, Japan

${ }^{2}$ Department of Surgery, Iwata City Hospital, Japan

${ }^{3}$ Department of Radiology, Hamamatsu University School of

Medicine, Japan

${ }^{4}$ Hamamatsu University School of Medicine, Japan

*Address for Correspondence

Takanori Hiraide, Second Department of Surgery, Hamamatsu University School of Medicine, 1-20-1 Handayama, Higashi-ku, Hamamatsu 4313192, Japan, Tel: +81-53-435-2279; Fax: +81-53-435-2273; E-mail: hiraide@hama-med.ac.jp

Submission: 12 April, 2017

Accepted: 10 May, 2017

Published: 20 May, 2017

Copyright: @ 2017 Hiraide T, et al. This is an open access article distributed under the Creative Commons Attribution License, which permits unrestricted use, distribution, and reproduction in any medium, provided the original work is properly cited.

reliable and indispensable treatment for patients with multiple liver metastases, hepatectomy remains the better therapeutic strategy for patients with a few hepatic foci $[5,6]$. However, hepatectomy is feasible in only $20-35 \%$ of patients with liver metastases. Additionally, most patients with Colorectal Cancer Liver Metastasis (CRLM) develop intrahepatic and/or extrahepatic recurrence even after curative hepatectomy [6]. Preoperative staging is important for selection of patients who can potentially undergo resection of CRLM. To identify the number and location of metastases, contrastenhanced Computed Tomography (CT) or Magnetic Resonance Imaging (MRI) of the liver is generally used. Abdominal and chest CTs are usually performed to examine extrahepatic disease. To sensitively identify the extrahepatic lesions, ${ }^{18} \mathrm{~F}$-Fluorodeoxyglucose (FDG) Positron Emission Tomography (FDG-PET) has been utilized in patients with CRLM [7]. FDG-PET can detect occult metastases and suspicious lesions that may be indeterminable with conventional radiological imaging $[8,9]$. When PET is combined with CT (PET/CT), functional and anatomical information is collected simultaneously. Some oncologists and surgeons have emphasized the usefulness of FDG-PET in patients with CRLM, however, FDG-PET fails to detect small lesions $(<1 \mathrm{~cm})$ due to poor spatial resolution, and preoperative FDG-PET is uninformative in $75 \%$ of patients with CRLM [10-12]. Considering the high cost of PET/CT examinations, it is unclear whether hepatectomy-candidates with CRLM should routinely undergo FDG-PET/CT [13,14].

In this study, we examined whether FDG-PET and PET/CT are useful as screening diagnostic modalities for patients with CRLM.

\section{Materials and Methods}

This study was approved by the Research Ethics Committee of the Hamamatsu University school of Medicine (IRB file No.: E15-320) and the methods were carried out in accordance with the approved 
Citation: Citation: Hiraide T, Sakaguchi T, Kikuchi H, Takeda M, Shibasaki Y, et al. The Role of Positron-Emission Tomography/Computed Tomography in Patients with Colorectal Liver Metastases who are Candidates for Liver Resection: Is it Useful?. J Surgery. 2017;5(1): 7.

ISSN: $2332-4139$

guidelines. Written informed consent was obtained from individual participants in accordance with the Declaration of Helsinki. All analyses of anonymous clinical patient data were performed retrospectively.

\section{The first term study}

Patients: Between January 2002 and December 2008, 50 patients with CRLM who were referred for hepatectomy underwent a standard diagnostic protocol that included enhanced chest and abdominal single-detector row CT and colonoscopy. FDG-PET was performed at the surgeons' discretion. Abdominal MRI was occasionally performed according to the radiologists' recommendation. As a result, 25 patients underwent FDG-PET during the preoperative workup (PET group), whereas 25 received conventional diagnostic procedures (control group). There was no perioperative mortality in either the PET or control group. The patients' characteristics are shown in Tables 1 and 2 .

Imaging protocol: Contrast-enhanced CT was conducted with two single-detector row CT scanners (CT HiSpeed Advantage or CT ProSpeed; GE Medical Systems, Milwaukee, WI, USA) using 10-mm collimation. Dynamic contrast material-enhanced CT was performed to assess intrahepatic lesions after intravenous administration of nonionic contrast media at an injection rate of $1.0 \mathrm{~mL} / \mathrm{s}$. MRI was performed using a 1.5-T MRI system (Signa Horizon EchoSpeed; GE Medical Systems, Milwaukee, WI, USA) with a phased-array coil. Gadodiamide hydrate was intravenously administered in dynamic MRI studies. FDG-PET was performed with a dedicated PET scanner (SHR-22000, Hamamatsu Photonics KK, Hamamatsu, Japan) at Hamamatsu PET Medical Examination Center. The serum glucose level measured at the time of 18F-FDG injection was $<150 \mathrm{mg} / \mathrm{dL}$ in all patients. After a six hour fast, the patient was hydrated with 500 $\mathrm{mL}$ of water, and 300 to $400 \mathrm{MBq}$ of FDG was intravenously injected. One hour later, emission images of the area between the base of the skull and proximal femora were acquired in a series of seven to eight positions ( $10 \mathrm{~min}$ per bed position). The images were corrected for attenuation and reconstructed using the ordered-subsets expectation maximization algorithm. Reconstructed images were displayed in coronal, transverse, and sagittal planes. Patients with diabetes mellitus were not excluded if their blood glucose levels were well controlled. When liver foci and extrahepatic lesions, if present, were resectable, CT angiography was performed to precisely diagnose the number and location of the hepatic lesions in both groups.

\section{The second term study}

Patients: Between January 2009 and December 2013, 45 consecutive patients were referred for hepatectomy. At the start of the second part of the study, we defined the criteria for performing PET (FDG-PET/CT) according to the results of the first term study: FDG-PET/CT was performed in patients showing three or higher Fong's clinical risk score (CRS), or in patients receiving neoadjuvant chemotherapy for CRLM [15]

Cases with obvious extrahepatic metastases detected by MDCT and MRI were not referred to our department in this term. Based this criteria, 45 patients were divided into "PET recommend" (PET group) ( $\mathrm{n}=30)$ and "PET not recommend" (non-PET group) $(\mathrm{n}=15)$ groups. Only one patient underwent a single row CT, since the Multi-
Detector row CT (MDCT) apparatus was not installed in our institute at that time. All except this patient underwent enhanced MDCT. MRI was performed in 43 of 45 patients excluding contraindications. Patient characteristics are shown in Table 3.

Imaging protocol: Contrast-enhanced CT was conducted using an Aquillion 64-MDCT scanner (Toshiba, Tokyo, Japan) with pre- and post-contrast triple-phase (arterial, portal venous, and equilibrium) scans after injection of $2-\mathrm{mL} / \mathrm{kg}$ nonionic iodine contrast media at an injection rate of $3.0 \mathrm{~mL} / \mathrm{s}$.

MRI was performed with a 1.5-T MRI system (Signa EXCITE Xl 1.5 T, GE Healthcare Japan) and a 3.0-T MRI system (Discovery MR750 3.0T, GE Healthcare Japan) with a 4-channel torso-array coil. Double echo T1-weighted (T1w) gradient-echo image (in-phase/ out-of-phase), respiratory triggered fat-saturated $\mathrm{T} 2$-weighted $(\mathrm{T} 2 \mathrm{w})$

Table 1: Patient's characteristics in the first term (2002-2008).

\begin{tabular}{|c|c|c|c|}
\hline & $\begin{array}{c}\text { PET Group } \\
(\text { with PET, } \\
\mathrm{n}=25)\end{array}$ & $\begin{array}{c}\text { Control Group } \\
(\text { without PET, } \\
\mathrm{n}=25)\end{array}$ & $\boldsymbol{P}$ value \\
\hline Sex male $:$ female & $18: 7$ & $17: 8$ & n.s. \\
\hline Mean age (years) & $62.0 \pm 8.3$ & $63.9 \pm 8.9$ & n.s. \\
\hline Time of liver metastasis & & & \\
\hline Synchronous & 7 & 5 & n.s. \\
\hline Metachronous & 18 & 20 & n.s. \\
\hline $1-6: 7-12: \geq 13$ months & $5: 2: 11$ & $6: 3: 11$ & \\
\hline Size in diameter & & & n.s. \\
\hline Mean, range (mm) & $36.1,(10-90)$ & $32.1,(15-70)$ & n.s. \\
\hline$\leq 5$ cm $:>5$ cm & $19: 6$ & $21: 4$ & n.s. \\
\hline Number of liver metastasis & & & 0.02 \\
\hline Solitary $:$ Multiple & $9: 16$ & $15: 10$ & \\
\hline Identified extrahepatic lesion & 6 & 0 & $0: 0: 0$ \\
\hline LN : bone $:$ Lung & $3: 1: 2$ & & \\
\hline
\end{tabular}

LN: lymph node, n.s.: not significant

Table 2: Fong's clinical risk scores for the first term (2002-2008).

\begin{tabular}{|c|c|c|c|c|c|}
\hline \multirow{2}{*}{ No. of case } & \multicolumn{4}{|c|}{ Clinical Risk Score (CRS) } & \multirow{2}{*}{} \\
\cline { 2 - 5 } & $\mathbf{0 - 1}$ & $\mathbf{2}$ & $\mathbf{3}$ & $\mathbf{4}$ & $\mathbf{5}$ \\
\hline PET Group (n=25) & & & & & \multirow{2}{*}{1} \\
\hline Hx-not performed & & & 4 & 1 & \\
\hline Due to extrahepatic lesion & & & & & \\
\hline Hx-performed & 10 & $2(1)$ & $6(5)$ & $1(1)$ & \\
\hline Recurrence after Hx & 7 & $1(1)$ & $4(3)$ & $1(1)$ & \\
\hline No recurrence after Hx & 3 & 1 & $2(2)$ & & \\
\hline $\begin{array}{c}\text { Change rate by PET work } \\
\text { up (\%) }\end{array}$ & 0 & 0 & 40 & 50 & \multirow{2}{*}{100} \\
\hline Control Group (n=25) & & & & & \\
\hline Hx-performed & 10 & $8(1)$ & $5(3)$ & $2(1)$ & \\
\hline $\begin{array}{c}\text { Recurrence after Hx } \\
\text { No recurrence after Hx }\end{array}$ & 5 & $4(1)$ & $4(2)$ & $2(1)$ & \\
\hline $\begin{array}{c}\text { Change rate by conventional } \\
\text { work up }\end{array}$ & 0 & 4 & $1(1)$ & & \\
\hline
\end{tabular}

$\mathrm{Hx}=$ Hepatectomy, $(\mathrm{)}$ = number of patients receiving neoadjuvant chemotherapy with multiple hepatic metastases Fong's clinical risk score: Five prognostics variables were assigned 1 point each: primary node positive, multiple liver foci, disease-free interval $<12$ months, largest hepatic tumor $>5 \mathrm{~cm}$, and CEA $>200$ $\mathrm{ng} / \mathrm{mL}$. The total score for each patient was obtained by addition. 
Citation: Citation: Hiraide T, Sakaguchi T, Kikuchi H, Takeda M, Shibasaki Y, et al. The Role of Positron-Emission Tomography/Computed Tomography in Patients with Colorectal Liver Metastases who are Candidates for Liver Resection: Is it Useful?. J Surgery. 2017;5(1): 7.

ISSN: $2332-4139$

image, three-dimensional gradient echo T1-weighted image, and Diffusion-Weighted Image (DWI) sequences were obtained before and after the intravenous administration of a contrast medium. A combination of T1-wieghted contrast-enhanced dynamic images were obtained at 25-35 s (arterial phase), 55-65 s (portal phase), 85-95 $\mathrm{s}$ (equilibrium phase), and $15 \mathrm{~min}$ (hepatobiliary phase) after bolus injection of $0.1-\mathrm{mL} / \mathrm{kg}$ gadolinium ethoxybenzyl diethylenetriamine pentaacetic acid (Gd-EOB-DTPA, Primovist, Bayer Schering Pharma, Berlin, Germany) followed by a $15-$ to $20-\mathrm{mL}$ saline flush. The injection rate was $2 \mathrm{~mL} / \mathrm{s}$.

FDG-PET/CT was performed with a True Point Biograph 16 device (Siemens Healthcare, Bayern, Germany) with an in-plane axial spatial resolution of $6.3 \mathrm{~mm}$ at full width at half maximum after fasting for more than six hours. The serum glucose concentrations measured before the ${ }^{18} \mathrm{~F}$-FDG injection were $<150 \mathrm{mg} / \mathrm{dL}$. In the patients with diabetes mellitus, these levels were $<200 \mathrm{mg} / \mathrm{dL}$. Image acquisition began approximately $60 \mathrm{~min}$ after an intravenous injection of approximately $4 \mathrm{MBq} / \mathrm{kg}$ of ${ }^{18} \mathrm{~F}$-FDG. Spiral CT images were acquired at $110 \mathrm{mAs}$ and $120 \mathrm{kVp}$ (current-time product) from the thigh to the head, followed by a three-dimensional craniocaudal PET scan. PET images were reconstructed using CT data with corrections for attenuation and reoriented into axial, sagittal, and coronal slices.

\section{Image Analysis}

All images were reviewed by our institute radiologists (more than 10 years of experience and board certified by the Japan Radiological Society).

\section{Follow-up}

After surgery, patients followed-up every three months for the first three years and every six months thereafter. At each visit, a history was obtained, physical examination was performed, and Carcinoembryonic Antigen (CEA) and carbohydrate antigen 19-9 (CA19-9) were measured. Contrast-enhanced CT scans of the chest,

Table 3: Patient's characteristics in the second term (2009-2013).

\begin{tabular}{|c|c|c|c|}
\hline & $\begin{array}{c}\text { PET } \\
\text { recommend }\end{array}$ & $\begin{array}{l}\text { PET not } \\
\text { recommend }\end{array}$ & $p$ value \\
\hline & $\begin{array}{c}\text { (with PET, } \\
\mathrm{n}=30 \text { ) }\end{array}$ & $\begin{array}{c}\text { (without PET, } \\
n=15)\end{array}$ & \\
\hline Sex male : female & $22: 8$ & $10: 5$ & n.s. \\
\hline Mean age (years) & $63.3 \pm 10.7$ & $65.3 \pm 10.3$ & n.s. \\
\hline \multicolumn{4}{|l|}{ Characterization } \\
\hline Mean CRS* & 2.07 & 1.18 & 0.001 \\
\hline number of $C R S \geq 3$ & 12 & 0 & \\
\hline Neoadjuvant chemotherapy & 17 & 0 & 0.002 \\
\hline Identified extrahepatic lesion\# & 6 & 0 & n.s. \\
\hline Local : Pelvis : Lung & $2: 1: 3$ & 0:0:0 & \\
\hline $\begin{array}{l}\text { Recurrence after } \\
\text { hepatectomy }\end{array}$ & 17 & 6 & n.s. \\
\hline Remnant liver & 12 & 4 & \\
\hline $\begin{array}{l}\text { Other lesion (local, bone, } \\
\text { lung) }\end{array}$ & 8 & 2 & \\
\hline \multicolumn{4}{|l|}{ Disease-free interval } \\
\hline $1-6: 7-12: \geq 13$ months & $9: 6: 2$ & $1: 0: 5$ & \\
\hline
\end{tabular}

${ }^{*}$ CRS: Fong's Clinical risk score,

\#: contains duplicate, n.s.: not significant abdomen, and pelvis, were performed every six months.

\section{Fong's CRS}

Characteristics of patients with CRLM were evaluated according to Fong's prognostic scoring system [15]. Five prognostics variables were assigned one point each: primary node positive, multiple liver foci, disease-free interval $<12$ months, largest hepatic tumor $>5 \mathrm{~cm}$, and CEA $>200 \mathrm{ng} / \mathrm{mL}$. CRS is a clearly defined and widely applicable score of clinical criteria for the selection of patients that are likely to benefit from resection of CRLM.

\section{Statistical analysis}

The differences in patient characteristics between the control and PET groups (the first part of the study), and the "PET recommend" and "PET not recommend" groups (the second part of the study) were examined using Student's $t$ test or chi-square test. To identify significant factors related to Overall Survival (OS) and Disease-Free Survival (DFS), the Kaplan-Meier product limit estimators were calculated and compared using log-rank tests. Clinicopathological variables of potential prognostic value were dichotomized and analyzed for their effect on DFS and OS. A p $<0.05$ was considered significant. The data were analyzed using the IBM SPSS Statistics software.

\section{Results}

Role of preoperative FDG-PET on the detection of extrahepatic lesions in the first term study

Between January 2002 and December 2008, 50 patients with CRLM who were referred for hepatectomy underwent a standard diagnostic protocol (CT and colonoscopy, Table 1). Among them, 25 patients (control group) did not undergo FDG-PET, after surgeons determined that extrahepatic diseases would be negligible. All 25 patients in the control group underwent hepatectomy after extrahepatic lesions were not identified under enhanced CT.

In contrast, hepatectomy was canceled in six (24\%) of the 25 PET group patients because FDG-PET revealed extrahepatic lesions (lymph nodes in para-aortic, peri-hepatic hilar, and mediastinal regions; pelvic bone metastasis; and multiple pulmonary metastases). All extrahepatic lesions were undetectable by contrast enhanced single detector-row CT; these lesions were visualized as hot spots under FDG-PET. These six patients underwent systemic chemotherapy thereafter.

\section{OS and DFS in the control and PET groups in the first term}

To investigate whether FDG-PET could influence treatment strategy or prognosis, we compared OS and DFS in between the control and PET groups. The median follow-up was 58 months (range 8-120). The OS of the six patients showing PET-positive extrahepatic lesions after radiologic screening was significantly worse than those of patients after hepatectomy (Figure 1a). The 5-year OS rates after hepatectomy in the PET $(n=19)$ and control $(n=25)$ groups were $60.5 \%$ and $46.0 \%$, respectively ( $p=0.33$; Figure $1 \mathrm{a}$ ). The 5 -year DFS rates were $15.8 \%$ and $26.0 \%$, respectively ( $p=0.45$; Figure $1 b)$. There were no statistically significant differences in OS and DFS rates between the two groups. 
Citation: Citation: Hiraide T, Sakaguchi T, Kikuchi H, Takeda M, Shibasaki Y, et al. The Role of Positron-Emission Tomography/Computed Tomography in Patients with Colorectal Liver Metastases who are Candidates for Liver Resection: Is it Useful?. J Surgery. 2017;5(1): 7.

ISSN: $2332-4139$

\section{Effective application of FDG-PET as a preoperative diagnostic modality and establishing the criteria for FDG-PET indication}

We examined whether patients with CRLM should undergo FDG-PET as a pre-hepatectomic diagnostic modality. Among the various parameters analyzed, we focused on Fong's CRS [15]. All 50 patients were categorized by Fong's CRS system, and the relationships between CRS and clinical course were evaluated (Table 2). All six patients with PET-positive extrahepatic lesions showed a CRS score of three or higher. Furthermore, OS was significantly worse in patients with CRSs $\geq 3$, than those with CRSs $\leq 2$ (low CRS) in both control and PET groups (Figure 2). These findings suggest that Fong's CRS may be helpful to detect extrahepatic lesions.

From these analyses, we decided to perform FDG-PET/CT only when the patients with CRLM showed a Fong's score of three or higher or when the patients had received neoadjuvant chemotherapy for multiple liver metastases.

\section{Verification of the PET criteria}

In the second term, 45 patients were referred to our department for hepatectomy. The median follow-up was 49 months (range 11-60). Based on our criteria, 30 and 15 patients were enrolled in either the "PET recommend" (PET group) or "PET not recommend" (non-PET group) groups, respectively (Table 3). In the PET group, hepatectomy was not performed in four (13\%) patients because the FDG-PET/CT revealed six extrahepatic lesions, including local recurrence, pelvic bone metastasis, and pulmonary metastases. These lesions were visualized as hot spots under FDG-PET/CT. Five of six extrahepatic lesions were detected by contrast enhanced MDCT or MRI. In the non-PET group, all patients underwent hepatectomy. All operations were completely successful and there was no perioperative mortality in either group.

Next, we investigated the prognosis after screening examinations for hepatectomy. After hepatectomy, recurrent foci were found under the follow-up enhanced CT in 17 of 26 in the PET patients and in six of 15 non-PET patients (Table 3). Although the recurrence rates did not significantly differ between the two groups, in the PET group, early recurrence (within six months after hepatectomy) was found in nine of 17 recurrent patients (53\%). In contrast, early recurrence in the non-PET group was found in one of six patients (17\%). The remaining five patients showed recurrent foci 13 months after hepatectomy. Among them, two of four cases showing remnant liveralone recurrence underwent repeat hepatectomy. The remaining two cases underwent systemic chemotherapy due to multiple liver metastases.

The 5-year OS rate after hepatectomy was $55.9 \%$ and $86.2 \%$ ( $p$ $=0.04)$, and the 5 -year DFS rate was $30.8 \%$ and $60.0 \%(p=0.02)$ in the PET and non-PET groups, respectively (Figure 3 ). Moreover, disease quickly recurred after hepatectomy in the PET group (median disease-free duration 10.5 months versus 60.0 months). The PET group showed poorer prognosis and higher recurrence rates, even if the PET/CT omitted the unnecessary hepatectomy by detecting the extrahepatic lesions.

The detection rates of extrahepatic lesion under PET/CT and CT
We examined the detection rates of intra- and extrahepatic lesion under PET/CT and enhanced CT in the PET group of the second term. For liver foci, the final diagnosis was based on the histological analysis of surgical specimens of resected livers. For the extrahepatic lesions, the final diagnosis was based on pathological or follow-up radiological findings.

We evaluated 74 in total lesions (68 intra- and six extrahepatic) in the PET group (Table 4). Among 68 intrahepatic foci, only one was undetectable under CT ( $98.5 \%$ sensitivity). This focus was pathologically identified in the resected hepatic specimen. By contrast, PET failed to detect 12 of 68 liver lesions ( $82.4 \%$ sensitivity; $p<0.01$ ). These 12 lesions were $<10 \mathrm{~mm}$ in diameter, suggesting the inability of PET to detect small lesions. Among the six extrahepatic

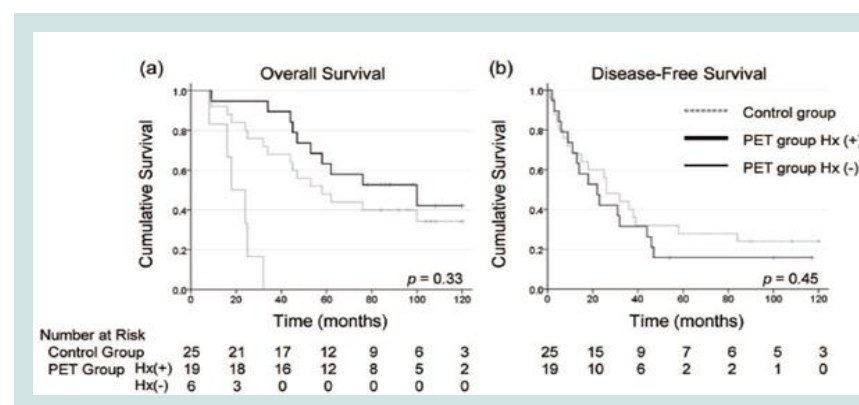

Figure 1: Overall and Disease-Free survivals in the first term study. Overall (a) and disease-free (b) survival curves of the patients in control group (dashed line, $n=25$ ), PET group with hepatectomy: $\mathrm{Hx}(+)$ (bold solid line, $n=19)$ and PET group without hepatectomy: $\mathrm{Hx}(-)$ (solid line, $n=6)$.

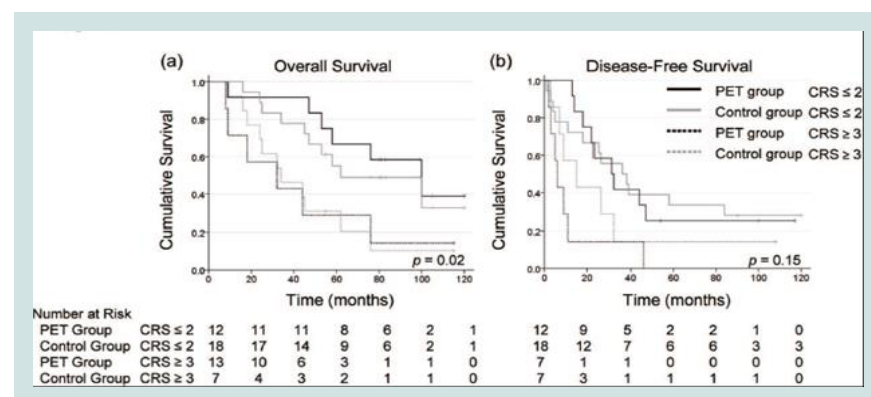

Figure 2: Overall and disease-free survivals stratified by CRS in the first term study. Overall (a) and disease-free (b) survival curves of the patients in control group (CRS $\leq 2$; solid line, $C R S \geq 3$; dashed line) and PET group (CRS $\leq 2$; bold solid line, $C R S \geq 3$; bold dashed line).

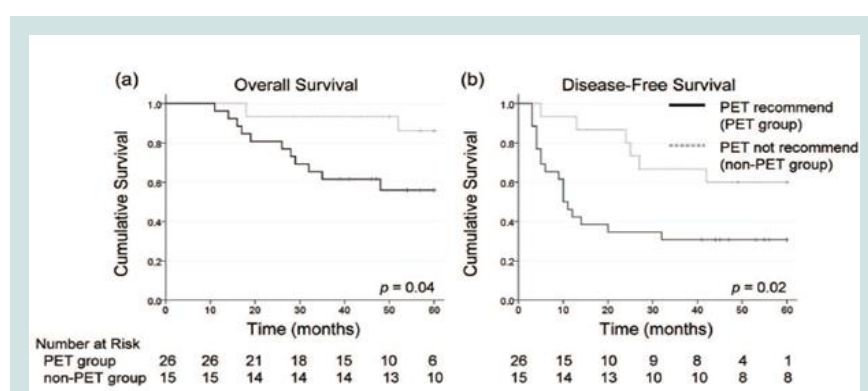

Figure 3: Prognoses in PET recommended and PET not recommended groups in the second term. Overall (a) and disease-free (b) survival curve of the patients in PET-recommended group (solid line, $n=26$ ) and PET not recommended group (dashed line, $n=15$ ). 
Citation: Citation: Hiraide T, Sakaguchi T, Kikuchi H, Takeda M, Shibasaki Y, et al. The Role of Positron-Emission Tomography/Computed Tomography in Patients with Colorectal Liver Metastases who are Candidates for Liver Resection: Is it Useful?. J Surgery. 2017;5(1): 7.

foci that were proved to be locally recurring or metastatic diseases by follow-up radiological findings, five foci were clearly depicted under enhanced MDCT. The patient with the only lesion detected by PET but not enhanced CT underwent single detector-row CT. Our data suggest that the combination of contrast enhanced MDCT and MRI is appropriately sensitive to detect small recurrent foci in hepatectomy candidates with CRLM.

\section{PET/CT is strongly affected by chemotherapy}

Systemic chemotherapy is a common treatment in patients with CRLM, however, the diagnostic accuracy of PET and PET/CT is strongly affected by chemotherapy [16]. The typical case in our series was as follows: the patient having rectal adenocarcinoma with simultaneous liver metastases had undergone Abdominoperineal Resection (APR). The preoperative enhanced CT did not detect the focus, other than the original (rectum) and hepatic foci. The patient received a neoadjuvant chemotherapeutic combination of 5-fluorouracil, leucovorin, and oxaliplatin (mFOLFOX6) and bevacizumab during eight months after surgery. Four weeks after the completion of the final chemotherapy course, MDCT and PET/ CT scans were performed as screening diagnostic modalities before hepatectomy. MDCT detected the hepatic metastatic lesion and osteolytic lesion in the pelvic bone (Figures $4 \mathrm{a}$ and $4 \mathrm{~b}$ ). The PET/CT did not show hot spots for either the hepatic or pelvic bone lesion

Table 4: Detection of intra- and extra-hepatic lesions under Conventional Modality (CT and MRI) and PET/CT in PET-recommend group.

\begin{tabular}{|c|c|c|c|c|c|}
\hline & $\begin{array}{c}\text { No. of } \\
\text { lesions }\end{array}$ & Detected & Undetected & Detected & Undetected \\
\cline { 2 - 6 } & & Under CM* & Under CM* & $\begin{array}{c}\text { Under } \\
\text { PET/CT }\end{array}$ & $\begin{array}{c}\text { Under PET/ } \\
\text { CT }\end{array}$ \\
\hline Intrahepatic & 68 & 67 & 1 & 56 & 12 \\
\hline Extrahepatic & 6 & 5 & 1 & 6 & NA \\
\hline Total & 74 & 72 & 2 & 62 & 12 \\
\hline & Intrahepatic & Extrahepatic & & & \\
\hline $\begin{array}{c}\text { CT and MRI } \\
\text { sensitivity }\end{array}$ & $98.5 \%$ & $83.3 \%$ & & & \\
\hline $\begin{array}{c}\text { PET/CT } \\
\text { sensitivity }\end{array}$ & $82.4 \%$ & $100 \%$ & & & \\
\hline
\end{tabular}

CM: Conventional Modality; NA: Not Assigned

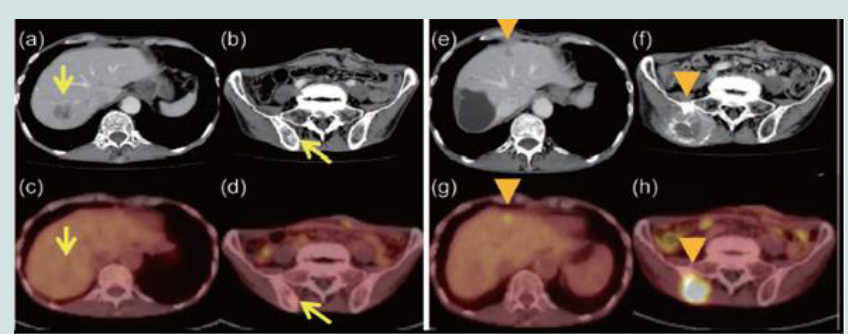

Figure 4: Chemotherapy's effect on 18F- fluorodeoxyglucose incorporation into metastatic foci under PET/CT. A 74-year-old man having rectal adenocarcinoma and simultaneous liver metastasis underwent abdominoperineal resection. Thereafter, he underwent FOLFOX-based chemotherapy for eight months. Four weeks after the completion of the final chemotherapy course enhanced abdominal CT revealed the hepatic metastasis in the right lobe and an osteolytic lesion in the pelvic bone $(\mathbf{a}, \mathbf{b})$. However, FDG-PET/CT did not show any hot spots in these lesions (arrow) $(\mathbf{c}, \mathbf{d})$. Since the pelvic bone lesion was negligible, he underwent partial hepatectomy. Three months after hepatectomy, both enhanced CT (e,f), and FDG-PET/CT $(\mathbf{g}, \mathbf{h})$ revealed the newly developed intrahepatic recurrence and pelvic bone metastasis (arrow head)
(Figures $4 \mathrm{c}$ and $4 \mathrm{~d}$ ). We concluded that the osteolytic lesion in the pelvic bone was negligible, and performed hepatectomy. Three months after hepatectomy, MDCT and PET/CT scans detected new metastatic foci in the remnant liver and obvious pelvic bone metastases (Figures $4 \mathrm{e}-4 \mathrm{~h}$ ). PET/CT may therefore not be a suitable screening diagnostic modality for CRLM patients receiving chemotherapy.

\section{Discussion}

Surgery is the primary curative treatment for recurrent and metastatic (mainly in the liver) Colorectal Cancer (CRC). Preoperative imaging plays an important role in the diagnosis and management of CRC patients. The most frequently used imaging modalities for detection of CRC metastases are US, CT, MRI, and FDG-PET/CT [17]. Specifically, MDCT and MRI are used as the standard modalities for preoperative diagnosis of intra- and/or extrahepatic metastases.

FDG-PET is more sensitive than enhanced CT in detecting extrahepatic disease, upstaging patients in $16-31.6 \%$ of cases according to the published literature [18-20]. Recently, oncologists and surgeons tend to perform PET/CT in every patient with CRLM as a routine examination for detecting liver or occult extrahepatic lesions. Consequently, the high cost of PET/CT examination placed an enormous financial burden on the national healthcare system. Although it is unnecessary to utilize PET/CT as a routine examination for all patients with CRLM, there are currently no criteria or guidelines for the selection of patients who should receive PET/CT. In this study, we investigated whether preoperative PET or PET/CT examinations could provide additional information for hepatectomy candidates with CRLM, and sought to define the patient cohort for which preoperative PET/CT should be employed.

The results of this retrospective study suggest that preoperative PET or PET/CT has no effect on OS or DFS, even after the selection criteria were stratified using Fong's CRS (Figure 3). Since the progress in the development of conventional detection modalities, such as enhanced abdominal MDCT and MRI, has resulted in high sensitivity that allows the detection of small liver metastases ( $10 \mathrm{~mm}$ or less) and extrahepatic metastatic disease, hepatic lesions that are unidentifiable with these techniques are becoming rare, reducing the applicability of $\mathrm{PET} / \mathrm{CT}$ [21]. In postoperative recurrence, nine (53\%) of the 17 "PET recommend" (PET group) patients showed early recurrence (within six months) (Table 3). By contrast, only one "PET not recommend" (non-PET group) patient (7\%) showed recurrence within six months, and the other five patients (93\%) had recurrences 13 months or later (Table 3). These findings may negate the importance of PET/CT scan before liver resection in hepatectomy-candidates.

A large number of previous reports have demonstrated the benefits of PET or PET/CT. By contrast, recently the clinical usefulness of PET/CT examination needs reevaluation considering multidisciplinary treatment with a focus on systemic chemotherapy. Akhurst et al. reported the possibility that chemotherapy decreases the uptake of FDG into CRLM [16]. Furthermore, a meta-analysis of 11 studies including 906 liver foci in 223 patients revealed that MRI appears to be the most appropriate imaging modality for preoperative assessment of patients with CRLM undergoing neoadjuvant chemotherapy [22]. The diagnostic accuracy of PET and PET/CT is, therefore, strongly affected by chemotherapy. The chemotherapy may have resulted in false-negative PET/CT findings, limiting the 
Citation: Citation: Hiraide T, Sakaguchi T, Kikuchi H, Takeda M, Shibasaki Y, et al. The Role of Positron-Emission Tomography/Computed Tomography in Patients with Colorectal Liver Metastases who are Candidates for Liver Resection: Is it Useful?. J Surgery. 2017;5(1): 7.

applicability of this technique.

Additionally, the potential benefits of PET/CT must be weighed against the risk of delaying the surgery to perform this procedure. However, PET/CT may be beneficial in terms of assessing multidisciplinary treatment efficacy on other malignant neoplasms. Recent studies have reported that PET/CT has significant predictive and/or prognostic power when used as an indicator of treatment response in various cancers, including oropharyngeal squamous cell carcinoma, esophageal cancer, head and neck squamous cell cancer, non-small cell lung cancer, breast cancer, and rectal cancer [2329]. At this point, we do not deny the usefulness of FDG-PET/CT; however, the effect of chemotherapy on incorporation of FDG into recurrent foci on FDG-PET/CT is worth alarm.

Many countries struggle to maintain high-quality health care within existing budgets. This is difficult due to increasing healthcare costs as a result of population aging and expenses associated with implementation of new therapies and technologies, including diagnostic and functional imaging methods [30,31]. According to the fiscal report of the Ministry of Health, Labor, and Welfare released in Japan in 2013, the national health expenditure has reached more than 390 billion US dollars, accounting for about $10 \%$ of gross domestic product, which increases every year. The national health expenditure is expected to increase in the future. We believe that the combination of contrast enhanced MDCT and MRI utilizing T1-weighted (T1w), T2-weighted (T2w), and Diffusion-Weighted Imaging (DWI) sequences obtained before and after the administration of intravenous Gadolinium Binding Contrast Agent (GBCA) is sufficient for examining hepatectomy candidates. Additionally, we are convinced that the most important aspect is to review all images by radiologists with years of experience. The low efficiency of PET/CT could lead to delays in the treatment and increased healthcare costs; thus, PET/CT may not be appropriate for every hepatectomy candidate with CRLM.

This study has some limitation mostly consisting in its relatively small sample size, retrospective design, and single-center conduction. Therefore, a large-scale prospective validation study is needed to confirm these results.

\section{Conclusion}

Preoperative imaging modalities are of paramount importance for surgeons as tools for selecting appropriate candidates for surgery and devising appropriate surgical strategies for complete eradication of metastatic disease. Patients with CRLM and high CRS have a particularly high risk of extrahepatic disease and early recurrence, and they should be carefully selected for surgery. This retrospective study demonstrated that FDG-PET/CT was not associated with improved OS or DFS, suggesting that FDG-PET/CT is not always useful. Moreover, performing PET/CT can lead to increased healthcare costs. We may need to refrain from conducting such unnecessary examinations in order to reduce the financial burden of health care.

\section{References}

1. Greening DW, Lee ST, Ji H, Simpson RJ, Rigopoulos A, et al. (2015) Molecular profiling of cetuximab and bevacizumab treatment of colorectal tumours reveals perturbations in metabolic and hypoxic response pathways. Oncotarget 6: 38166-38180.

2. Long N, Moore MA, Chen W, Gao CM, Lai MS, et al. (2010) Cancer epidemiology and control in north-East Asia - past, present and future. Asian Pac J Cancer Prev 11 Suppl 2: 107-148.

3. Matsuda T, Marugame T, Kamo K, Katanoda K, Ajiki W, et al. (2010) Cancer incidence and incidence rates in Japan in 2004: based on data from 14 population-based cancer registries in the Monitoring of Cancer Incidence in Japan (MCIJ) Project. Jpn J Clin Oncol 40: 1192-1200.

4. Abdel-Nabi H, Doerr RJ, Lamonica DM, Cronin VR, Galantowicz PJ, et al. (1998) Staging of primary colorectal carcinomas with fluorine-18 fluorodeoxyglucose whole-body PET: correlation with histopathologic and CT findings. Radiology 206: 755-760.

5. Nakamura S, Suzuki S, Konno H (1999) Resection of hepatic metastases of colorectal carcinoma: 20 years' experience. J Hepatobiliary Pancreat Surg 6: 16-22.

6. Vigano L, Ferrero A, Lo Tesoriere R, Capussotti L (2008) Liver surgery for colorectal metastases: results after 10 years of follow-up. Long-term survivors, late recurrences, and prognostic role of morbidity. Ann Surg Oncol 15: 2458-2464.

7. Ruers TJ, Wiering B, van der Sijp JR, Roumen RM, de Jong KP, et al. (2009) Improved selection of patients for hepatic surgery of colorectal liver metastases with (18)F-FDG PET: a randomized study. J Nucl Med 50: 1036 1041.

8. Malik HZ, Gomez D, Wong V, Al-Mukthar A, Toogood GJ, et al. (2007) Predictors of early disease recurrence following hepatic resection for colorectal cancer metastasis. Eur J Surg Oncol 33: 1003-1009.

9. Wiering B, Krabbe PF, Dekker HM, Oyen WJ, Ruers TJ (2007) The role of FDG-PET in the selection of patients with colorectal liver metastases. Ann Surg Oncol 14: 771-779.

10. Fernandez FG, Drebin JA, Linehan DC, Dehdashti F, Siegel BA, et al. (2004) Five-year survival after resection of hepatic metastases from colorectal cancer in patients screened by positron emission tomography with F-18 fluorodeoxyglucose (FDG-PET). Ann Surg 240: 438-450.

11. Strasberg SM, Dehdashti F, Siegel BA, Drebin JA, Linehan D (2001) Survival of patients evaluated by FDG-PET before hepatic resection for metastatic colorectal carcinoma: a prospective database study. Ann Surg 233: 293-299.

12. Zubeldia JM, Bednarczyk EM, Baker JG, Nabi HA (2005) The economic impact of 18FDG positron emission tomography in the surgical management of colorectal cancer with hepatic metastases. Cancer biother Radiopharm 20: $450-456$

13. Lubezky N, Metser U, Geva R, Nakache R, Shmueli E, et al. (2007) The role and limitations of 18-fluoro-2-deoxy-D-glucose positron emission tomography (FDG-PET) scan and computerized tomography (CT) in restaging patients with hepatic colorectal metastases following neoadjuvant chemotherapy: comparison with operative and pathological findings. J Gastrointest Surg 11: 472-478.

14. Moulton CA, Gu CS, Law CH, Tandan VR, Hart R, et al. (2014) Effect of PET before liver resection on surgical management for colorectal adenocarcinoma metastases: a randomized clinical trial. JAMA 311: 1863-1869.

15. Fong Y, Fortner J, Sun RL, Brennan MF, Blumgart LH (1999) Clinical score for predicting recurrence after hepatic resection for metastatic colorectal cancer: analysis of 1001 consecutive cases. Ann Surg 230: 309-321.

16. Akhurst T, Kates TJ, Mazumdar M, Yeung H, Riedel ER, et al. (2005) Recent chemotherapy reduces the sensitivity of [18F]fluorodeoxyglucose positron emission tomography in the detection of colorectal metastases. J Clin Oncol 23: 8713-8716.

17. Bipat S, Niekel MC, Comans EF, Nio CY, Bemelman WA, et al. (2012) Imaging modalities for the staging of patients with colorectal cancer. Neth J Med 70: 26-34.

18. Arulampalam TH, Francis DL, Visvikis D, Taylor I, Ell PJ (2004) FDG-PET for the pre-operative evaluation of colorectal liver metastases. Eur J Surg Oncol 30: 286-291.

19. Kantorova I, Lipska L, Belohlavek O, Visokai V, Trubaĉ M, et al. (2003) Routine (18)F-FDG PET preoperative staging of colorectal cancer: comparison with conventional staging and its impact on treatment decision 
Citation: Citation: Hiraide T, Sakaguchi T, Kikuchi H, Takeda M, Shibasaki Y, et al. The Role of Positron-Emission Tomography/Computed Tomography in Patients with Colorectal Liver Metastases who are Candidates for Liver Resection: Is it Useful?. J Surgery. 2017;5(1): 7.

making. J Nucl Med 44: 1784-1788.

20. Wiering B, Krabbe PF, Jager GJ, Oyen WJ, Ruers TJ (2005) The impact of fluor-18-deoxyglucose-positron emission tomography in the management of colorectal liver metastases. Cancer 104: 2658-2670.

21. Seo HJ, Kim MJ, Lee JD, Chung WS, Kim YE (2011) Gadoxetate disodiumenhanced magnetic resonance imaging versus contrast-enhanced 18F-fluorodeoxyglucose positron emission tomography/computed tomography for the detection of colorectal liver metastases. Invest Radio 46: 548-555.

22. van Kessel CS, Buckens CF, van den Bosch MA, van Leeuwen MS, van Hillegersberg R, et al. (2012) Preoperative imaging of colorectal liver metastases after neoadjuvant chemotherapy: a meta-analysis. Ann Surg Oncol 19: 2805-2813.

23. Garsa AA, Chang AJ, Dewees T, Spencer CR, Adkins DR, et al. (2013) Prognostic value of F-FDG PET metabolic parameters in oropharyngeal squamous cell carcinoma. J Radiat Oncol 2: 27-34.

24. Ishihara R, Yamamoto S, Ishii H, Nagai K, Matui F, et al. (2012) Predicting the effects of chemoradiotherapy for squamous cell carcinoma of the esophagus by induction chemotherapy response assessed by positron emission tomography: toward PET-response-guided selection of chemoradiotherapy or esophagectomy. Int J Clin Oncol 17: 225-232.

25. Brun E, Kjellen E, Tennvall J, Ohlsson T, Sandell A, et al. (2002) FDG PET studies during treatment: prediction of therapy outcome in head and neck squamous cell carcinoma. Head Neck 24: 127-135.

26. Mac Manus MP, Hicks RJ, Matthews JP, McKenzie A, Rischin D, et al. (2003) Positron emission tomography is superior to computed tomography scanning for response-assessment after radical radiotherapy or chemoradiotherapy in patients with non-small-cell lung cancer. J Clin Oncol 21: 1285-1292.

27. Ogino K, Nakajima M, Kakuta M, Hayashi M, Yamaguchi S, et al. (2014) Utility of FDG-PET/CT in the evaluation of the response of locally advanced breast cancer to neoadjuvant chemotherapy. Int Surg 99: 309-318.

28. Nishimura J, Hasegawa J, Ogawa $Y$, Miwa H, Uemura M, et al. (2016) 18F-Fluorodeoxyglucose positron emission tomography (18F-FDG PET) for the early detection of response to neoadjuvant chemotherapy for locally advanced rectal cancer. Surg Today 46: 1152-1158.

29. Guillem JG, Moore HG, Akhurst T, Klimstra DS, Ruo L, et al. (2004) Sequential preoperative fluorodeoxyglucose-positron emission tomography assessment of response to preoperative chemoradiation: a means for determining longterm outcomes of rectal cancer. J Am Coll Surg 199: 1-7.

30. Hu YY, Kwok AC, Jiang W, Taback N, Loggers ET, et al. (2012) High-cost imaging in elderly patients with stage IV cancer. J Natl Cancer Inst 104: 1164-1172.

31. Rowan K (2008) Rising costs of medical imaging spur debate. J Natl Cancer Inst 100: 1665-1667. 